\title{
CRISPR/Cas9 Technology: Making Possibilities against Breast Cancer
}

\author{
V. Sanjuvikasini ${ }^{1}$, S. Shruthi ${ }^{1}$, M. Vijay Pradhap Singh ${ }^{2 *}$ \\ 1. Student, Vivekanandha College of Engineering for Women (Autonomous), Elayampalayam, Tiruchengode- 637 205, Namakkal, Tamilnadu, India. \\ 2*. Assistant Professor, Department of Biotechnology, Vivekanandha College of Engineering for Women (Autonomous), Elayampalayam, Tiruchengode, \\ Namakkal, Tamilnadu, India. \\ *Corresponding author's E-mail: vijaypradhapsingh@gmail.com
}

Received: 11-09-2020; Revised: 24-11-2020; Accepted: 02-12-2020; Published on: 15-12-2020. \begin{abstract}
Breast cancer initiation and progression underlies by mutation process which initiates in lobules and promotes metastasis. Genomes are the exact determination to explain cancer etiology. Survey says about nearly $92 \%$ of females in the U.S.A. are affected by deadly breast tumors during 2012-2016. The ability to correct such mutations holds huge promise for battling cancer. Recently, because of its great capabilities and accuracy, the CRISPR-Cas9 leading genome editing technique has been extensively used in the therapeutic examination of cancers. A novel tool appearance paved a way for scientists to carry out extensive treatments. CRISPR/Cas editing and developing techniques were first discovered in 1987 by Yoshizum Ishino and his colleagues. These techniques are used for drug discovery and development for formidable diseases. Being a big revolutionary technique, this is used widely to treat dangerous malignant breast cancer. We have discussed the role of CRISPR to fight against oncology infections, described the clear view of breast cancer diagnosis and treatment. A potential therapeutic CRISPR/Cas role in cancer therapy was proposed.
\end{abstract}

Keywords: Breast cancer, CRISPR/Cas9 technology, BRCA1, Mechanism, Application.

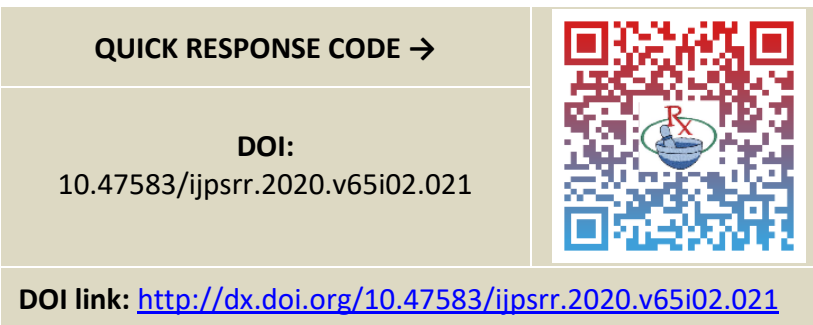

\section{INTRODUCTION}

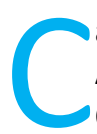

ancer is the leading cause of death worldwide. According to a survey by the World Health Organization (WHO), in 203023.6 million new cases will arise. Since it is a frightening disease, there is no particular vaccine or treatment to cure completely. Knowledge of somatic alternatives in breast cancer can be gained through studies on the genetic sequence ${ }^{1}$.

Breast cancer is the most threatening disease in both sexes. However, the majority of females are coming in with breast cancer while in men is quite less. Nulliparous women have a severe impact when compared to parous women. Studies show that they help prevent cancer by stopping the growth of free radicals in our environment. In 2018, 266,120 new breast cancer cases were reported; of these, 40,920 cases were fatal. Breast cancer is classified according to the over-expression of the cancer cell membrane, which includes hormone receptor Progesterone (PR), Estrogens (ER), and Human Epithelial growth factor Receptor (HER2). Based on these, breast cancer became divided into triple-positive breast cancer and triple-negative breast cancer. The patients with PR-, ER-, HER2- positive receptors are called triple-positive breast cancer, and the patients with negative receptors are called triple-negative receptors ${ }^{2}$.

The common and foregoing methods is to treat cancer are Surgery (mastectomy or lumpectomy, depending on the virility of the tumor cells), Chemotherapy (the use of drugs to kill the tumor cells), and Radiotherapy (introduction of high radio waves to kill the tumor cells). Chemotherapy is one of the most common methods used to treat tumor cells, but the studies proved that this method causes an unbearable side effect on the human being ${ }^{3}$.

Imagine being able to cure any genetic disease, prevent bacteria without antibiotics, and replace mosquitoes so that they can successfully spread malaria or cancer or transplant animal organs without rejection. The molecular machines to achieve these goals are not in the sci-fi novel set in the distant future. These are the goals achieved by the family of DNA sequences called CRISPR. As a gift of nature, in 1987, a Clustered Regularly Interspaced Short Palindromic Repeats, now commonly known as CRISPR were found in the immune system of Escherichia coli, a recurrence of genes 29 nucleotide series along with 32 nucleotides variable sequence fraction ${ }^{4}$.

In familiar usage, CRISPR (Pronounced "crispr") is shorthand for CRISPR/Cas9 system. CRISPR are specialized stretches of DNA. The protein Cas9 (or "CRISPR associated) is an enzyme that acts as molecular scissors, successfully slicing the strands of DNA. Based on the structure and sequence of nuclear proteins, the CRISPR/Cas9 bacterial adaptive immunity system became divided into three different types. There are:

\section{CRISPR/Cas type I}


2. CRISPR/Cas type II, and

3. CRISPR/Cas type III.

Of these, CRISPR/Cas type II is beneficial and works out but type I and type III was unfit for genome editing because of their complex structures. The CRISPR/Cas II system helps to identify and inhibits the viral DNA in the bacterial system. Three main molecules play an effective role in viral DNA modification is listed as

\section{Cas9 protein}

\section{CRISPR RNA}

3. Transactivating RNA.

Type II CRISPR helps to eliminate the viral sequence in three stages, phase I is the acquisition of the CRISPR/Cas system, during which it initially detects the viral sequence of DNA. The second stage is CrRNA biogenesis, in which the targeted viral compounds bind to the CRISPR complex. Finally, the CRISPR complex attacks DNA and degrades the occupational space with a single incision ${ }^{5}$. Recently, CRISPR/Cas system is most widely used in cancer research to diagnose the position of cancer and to correct it.

Here, we introduce the CRISPR self mechanism, detail about breast cancer diagnosis and treatment, timeline on CRISPR/Cas9 technology, the role of CRISPR in breast cancer, and its applications and advantages.

\section{Investigation of Breast Cancer}

The ancient Egyptian book Edwin Smith reported 8 cases of papyrus breast cancer or breast ulcers. In the early 1880 s, mastectomy was used to treat patients with breast cancer. Lumpectomy was first introduced in the 1980s, later developed, and in 1985 the advanced lumpectomy was developed; used before systematic adaptive therapy ${ }^{6}$. Chemotherapy is a well-known method of treating cancer, and many therapies have been started since then. Breast cancer is caused by genetic variations such as mutations, recombination, genetic migration, environmental influences, and physical changes.

Great technology has been developed to diagnose breast tumors, some of which have been discussed: Mammography is the most effective way to detect the presence of tumor cells in the human body, but fail (or not) to detect when the tumor cells are dense or mutated. Only an expert can handle this. Careless handling of it by specialists can lead to unnecessary surgery. Fluorescence In Situ Hybridization (FISH) helps to map specific cells in the breast, to determine if cells have mirror copies of the HER2 gene, and to distinguish between individuals with positive and negative cases. Experts tested the enzyme-linked immunosuppressant (ELISA) using two antigen sandwich ELISAs to identify tumor-affected areas. Here are some common ways to diagnose breast cancer ${ }^{7,8,9}$.

Dennis et al. concluded that, along with transtuzmub, have shown that chemotherapy is effective in treating $\mathrm{HER} /$ positive metastatic cancer and reducing mortality ${ }^{10}$.
Joseph et al. concluded and showed the great result in chemotherapy with concentrations of cyclophosphamide, methotrexate, and fluorouracil ${ }^{11}$. Gunter et al., studies have shown the impact of Pathological Complete Response (PCR) after neoadjuvant therapy for breast cancer. Anthra cyclins and taxanes were used for selected patients with intrinsic breast cancer. This therapy showed a positive result in patients with HER2 positive (non-luminal), luminal $\mathrm{B} / \mathrm{HER} 2-$ negative tumors. It has shown alternative results for luminal and non-luminal tumours ${ }^{12}$.

D.L. Hershman et al., Conducted experiments based on consensual and persistent hormone therapy. Hormone therapy includes Tamoxifer and Aromatase inhibitor (Al). Of the 8,769 women, only $49 \%$ of patients underwent fulltime hormone therapy. Their studies concluded that there was an increase in mortality from continuous and noncompliant hormone therapy ${ }^{13}$.

\section{TIMELINE ON CRISPR/Cas9 TECHNOLOGY}

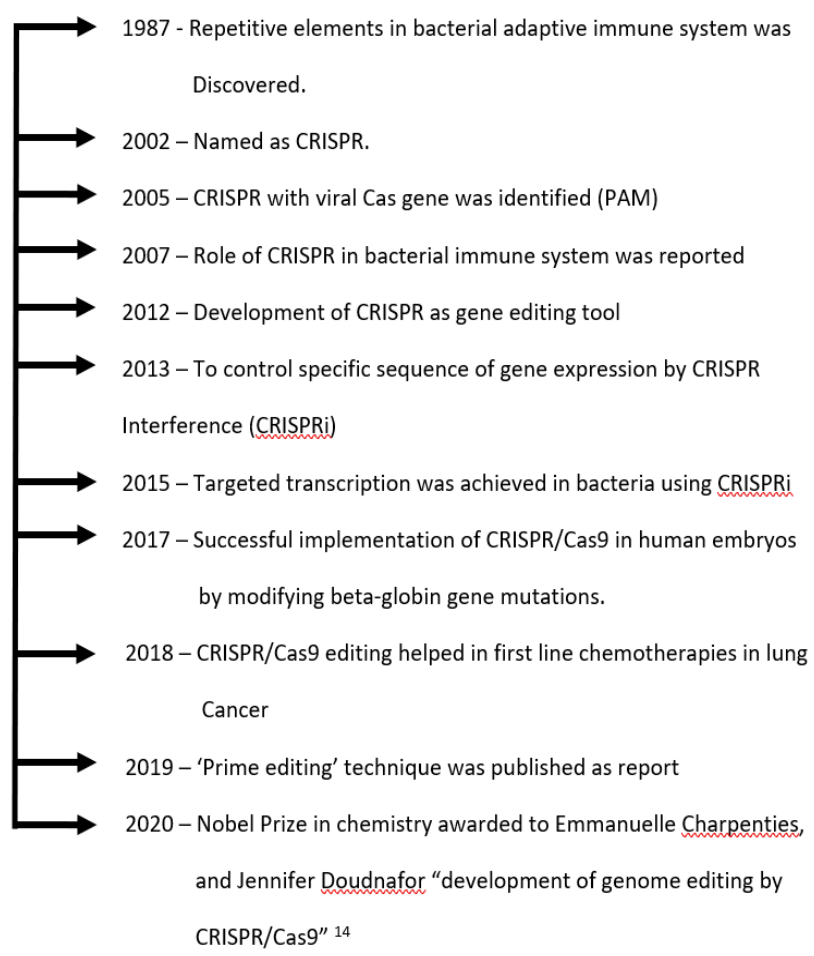

\section{Self-mechanism of CRISPR/Cas9 Technology}

CRISPR/Cas editing and developing techniques were first discovered in 1987 by Yoshizum Ishino and his colleagues. The genome of the different organism consists of a series of messages and instructions within their sequence of DNA. Genomic editing is involved to make changes the series of sequence to get changes in the instructions. It is performed by adding or deleting the DNA sequences and tricking the cells natural DNA repair mechanism into introducing the changes one wants. CRISPR/Cas9 provides the same.

The CRISPR Cas9 system, which is found in the adaptive immune system of bacteria, can regulate the genetic materials through three processes, called adaptation, expression, and interaction. The adoption phase is 
classified under the semi-independent subsystem, while the expression and intervention phases are classified under the executive subsystem ${ }^{15}$. The Cas9 protein helps to identify the cleavage target site of DNA. This Cas9 is made up of 6 domains namely (1) REC I (recognition lobe) (2) REC II (3) Bridge helix (4) PAM interacting (5) HNH and (6) RuvC ${ }^{16}$.

The CRISPR mainly contains CRISPR RNA (crRNA) and transactivating RNA (trancr RNA). Those help to cleave the targeted site of DNA. Cas 9 will be activated by using sgRNA (single guide RNA) which recognizes the Protospace Adjacent Motif (PAM) at the end of DNA where doublestrand gets unwind. PAM acts as a marker tool to differentiate the target site. After unwinding the doublestrand DNA, the crRNA cleaves or breaks off the target sequence, and then the target gets disintegrated by the system ${ }^{17}$.

The sgRNA fuses with crRNA and transRNA to improve target specification. The cas9 endocrinology is directed with the help of crRNA, which leads to a complementary conclusion in the targeted sequence. This site will be conquered by the cas9 protein where the PAM site acts as a catalytic site. The cas9 is directed to the DNA sequence to makes Double-Strand Break (DSB) at the targeted site and the gene is modified by either one of the two repair mechanisms.

DSB repairs non-homologous end joints (NHJ) pathways and frameshift mutations. . The NHEJ path is blocked by linking the reading frame of the coding sequences to the sys-regulatory components. It helps to eliminate the target gene and provide a disturbed reading framework. The next step is Homology Direct Repair (HDR), which consists of the sister chromatids of phase $S$ and phase $\mathrm{G} 2$, removing the targeted sequence and filling the deleted sequence with the desired exogenous sequence ${ }^{18}$.

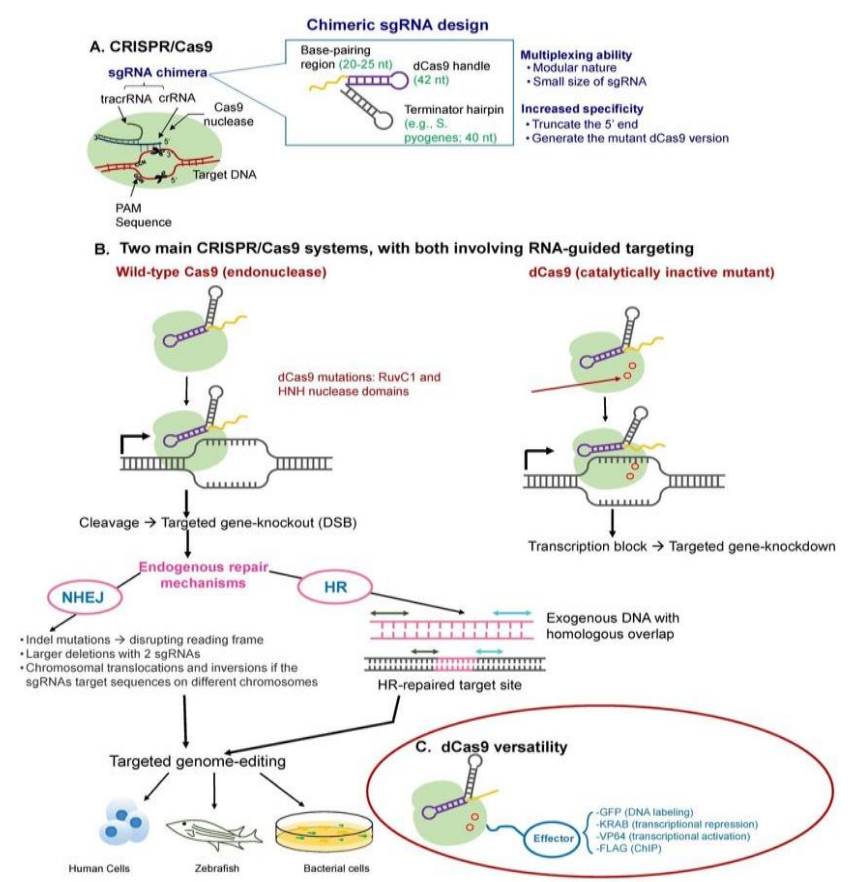

Figure 1: Action of CRISPR\Cas9 system ${ }^{18}$
CRISPR Cas9 plays an interesting role in gene therapy, in both cell lines and a mammal, shorting out monogenic disease; induce protective mutations, helps in CRISPRmediated cancer gene therapy ${ }^{19}$. CRISPR toolbox plays an exclusive role in gene silencing, with the use of CRISPR STOP a cluster of genes and transcripts were targeted ${ }^{20}$. This CRISPR toolbox of genetic management helps in mutation. For example, Oriza sativa GBSSI used as a target for mutation using the CRISPR\Cas9 ${ }^{21}$. This is effectively used in transcriptome analysis, which enables targeted nucleotide alternations ${ }^{22}$.

CRISPR, a natural tool, plays a broader role. Many ideological discussions, laws, and ethics are being developed because of CRISPR. It has played a key role in the treatment of various diseases and recent years the demolition of the gene, although interesting things about the future of CRISPR continue to bombard us in the years to come. Thus, CRISPR plays a major role in various fields as a developing tool in genetics, which has had a huge impact and revolutionized the biosphere.

\section{Role of CRISPR in Breast Carcinoma}

The CRISPR / Cas9 gene creates a favourable environment for genetic modification; Creates plasmid vectors and validates genetic algorithms in breast cancer treatment. Studies show that it is the best output technology for treating breast carcinoma.

Studies with TS genes are discussed. Human organoids from human epithelial subsets are classified based on the expression of two proteins, CD49F and APCM. The most commonly used mutated TS genes are p53, PTEN, and RB1. The sgRNA presented by the CAS9 system targets three TS genes. P53 is subject to nutline-3A, while PTEN and RB1 perform immunoblot analyzes. As P53 did not degrade, genetic modification occurred. To find out the capabilities of CRISPR / Cas9 editing, the next-generation sequencing was followed by transduction. CRISPR/Cas9 is simultaneous or sequentially knocks out the TS function in breast tumorigenesis ${ }^{23}$.

Studies on triple-negative cancer with PARP1 inhibition have been described. Cell lines with BRCA1 wild type and $B R C m$, inhibitory poly (ADP) -ribose polymerase (PAPR1) are selected from triple-negative cancer patients. The CRISPR device was introduced to inhibit the single gene (PAPR1) that produces modified cell lines. The CHOPCHOP algorithm was selected to initiate SgRNA and target PAPR1. The cluster of the three gRNA is synchronized based on the capabilities and off-targeting effects. Although gRNA2 and gRNA3 reached $82 \%$ efficiency in PAPR1 failure, gRNA1 efficiency reached $23 \%$. Therefore, gRNA1 was cloned using some protocols followed by the researchers. This method of targeting BARC wild type and BARCm type using inhibitor PAPR1 with CRSPR device potential has shown a positive effect on breast cancer treatment ${ }^{24}$. 


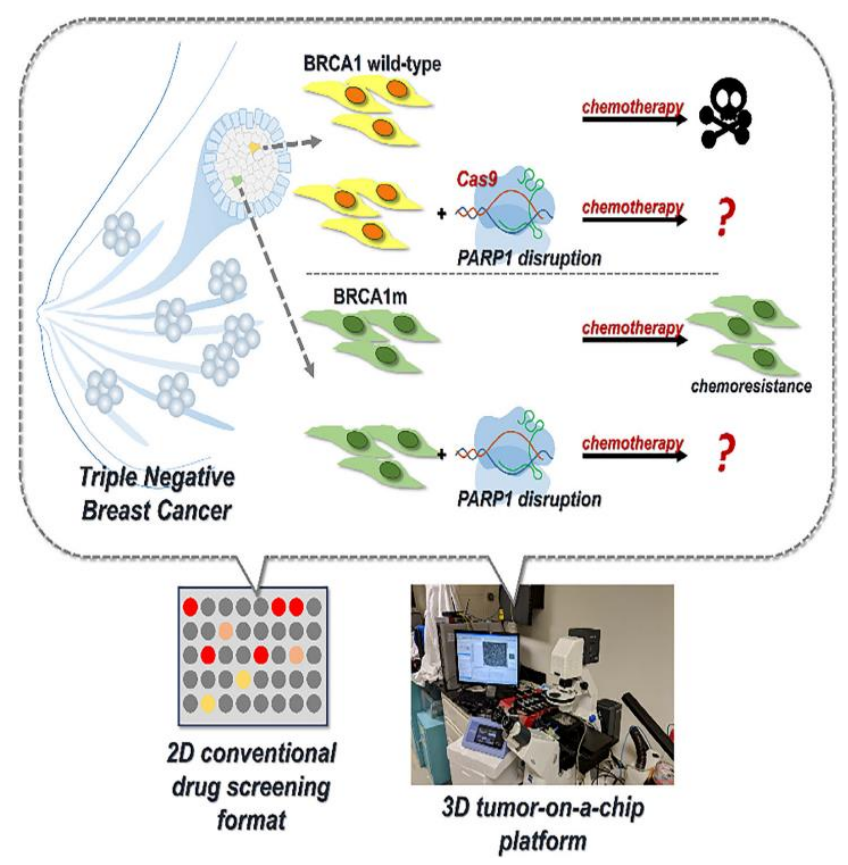

Figure 2: CRISPR- mechanism of breast cancer ${ }^{24}$

\section{Prize of CRISPR- Cancer}

In gene therapy, CRISPR/ Cas9 technology for all kinds of human diseases is impracticable in a single review. One of the most advanced technology applied several ways in the therapeutic process. CRISPR/ Cas9 shown possible in tumor therapy as ingenerate the tumor suppressor expression and inactivate the tumorous virus, as well as correct the mutation in monogenic diseases ${ }^{25}$.

Currently, CRISPR technology can protect people from cancer and examine the genetic makeup in all areas except cancer. Over the past few years, CRISPR / Cas9 have played an important role in the diagnosis and treatment of breast cancer and in the development of drug resistance to tumor cells. It will become an extensive tool in the field of therapy and accelerate cancer research. For example, Imatinib was targeting BCR-ABC1 synthesis in leukemia, Vermurafenib targeting BRAF $V 6000 \mathrm{E}$ in melanoma, and oxymeritinib EGFR in lung cancer ${ }^{26}$.

A research article on PubMed in 2019 found a huge increase in 9330 publications related to cancer research. Approximately $8.6 \%$ of people in this survey are associated with cancer. It is mainly found in leukemia, lung cancer and close to $26 \%$ of breast cancers. Model development, drug evaluation and cancer research are the focus of many researchers ${ }^{27}$.

In In-vivo and In-vitro gene delivery systems, it is difficult to pack large Cas 9 protein into low immunogenic AAV vectors. The isolation of the Cas 9 enzyme from $S$. pyogenes and $S$. aureus can cause certain infections in humans. Modifying the enzyme-forming strategy by using different types of bacterial proteins will overcome this defect. Although many challenges remain, the CRISPR / Cas9 system will have a major impact on genetic modification to improve patient outcomes in the future ${ }^{28}$.

\section{Significance of CRISPR/Cas9 Technology}

The CRISPR / Cas9 practice system plays an important role in cancer treatment in many ways. It is used to model mice for tumor disease. Cas9 genetic modification technology helps modify T-cells and gathers patients immunity to work against cancerous carcinoma ${ }^{29}$. CRISPR device helps CRISPR-based screening techniques on the widescreen help identify the required genes and drug targets. The myeloid leukemia tumor suppressor ASXL1 was converted to a mouse model using the Cas 9 devices. This helped to increase the lifespan of the rat $^{30}$. The main failure of chemotherapy treatment is the ability of tumor cells to fight chemotherapy drugs.

CRISPR Cas9 paves the way for the inactivation of drugresistant genes in tumor cells, increasing the potential of genes in tumor cells, and enhancing the effectiveness of chemotherapy $^{31}$. CRISPR / CAS9 sometimes cause offtarget mutations, leading to a risk factor. The CRISPR / Cas9 system should be carefully designed to avoid this risk. Guide RNA could not understand some molecular mechanisms. Online tools can help you find off-targets and capabilities. Cech and his colleagues introduced the "popin/pop-up" approach to reducing the efficiency of CRISPR / $\mathrm{CAS9}^{32}$. Compared to other engineering nucleases such as ZFNs and TALENs, CRISPR / Cas9 have higher target efficiency, lower cost, and easier testing.

\section{CONCLUSION}

CRISPR/Cas9 an engineering technique is playing a major role in the biological field, providing new alternations in an easy way for various disease treatments; any organism's genome can be easily modified. As a part, the CRISPR toolbox in breast cancer research has a great potential to treat tumor cells efficiently. The researchers say genomewide specifications should be improved in the CRISPR technique. Being a developing and most preferable tool, it takes a major place in research field to diagnose and treat diseases, in gene-editing and gene therapy. This method of gene-editing technique is a simple process that can be carried out easily. The development of the CRISPR/Cas9 system from the basic level of the clinical method shows a powerful way in treating drug-resistant. With the potential of the CRISPR/Cas9 system, it can easily overcome the complexity of cancer and gives a suitable cancer drugresistant. The future with CRISPR has a great impact in the world.

From the knowledge of the above review, we conclude that even numerous old and present strategies are being used to treat breast cancer; there is reducing in mortality rate. By introducing the CRISPR gene editing method, demonstrated preferable execution over different treatments and medicines. It can prevent more mortality rate than other treatments.

Subsequently, we sum up yet there is a particular antitumor drug for complete cure from breast cancer. From the experimental results, the CRISPR technique can act effectively than different methods. 


\section{REFERENCES}

1. Stefano Annunziato, Catrin Lutz, Linda Henneman, Jinhyuk Bhin, Kim Wong, Bjørn Siteur, Bas van Gerwen, Renske de Korte-Grimmerink, Maria Paz Zafra, Emma M Schatoff, Anne Paulien Drenth, Eline van der Burg, Timo Eijkman, Siddhartha Mukherjee, Katharina Boroviak , Lodewyk FA Wessels, Marieke van de Ven, Ivo J Huijbers, David J Adams, Lukas E Dow, \& Jos Jonkers, In situ CRISPR-Cas9 base editing for the development of genetically engineered mouse models of breast cancer, The EMBO Journal, 2020, 39:e102169, https://doi.org/10.15252/embj.2019102169

2. Phuong Tran, Sang-Eun Lee, Dong-Hyun Kim, Yong-Chul Pyo, Jeong-Sook Park, Recent advances of nanotechnology for the delivery of anticancer drugs for breast cancer treatment, Journal of Pharmaceutical Investigation, 2019, ISSN 2093-6214. https://doi.org/10.1007/s40005-019-00459-7

3. Ghulam Akbar, Muhammad Anjum Zia, Faiz Ahmad Joyia, Ali Ahmad, Sana Ahmad, Neha Arooj, Muhammad Muzammal Saeed, CRISPR Cas9: Making Progress Against Cancer, Journal of Environmental\& Agricultural Sciences, 22(2), 2020, 44-57.

4. Xin Xiong, Meng Chen, Wendell A. Lim, Dehua Zhao, and Lei S. Qi, CRISPR/Cas9 for Human Genome Engineering and Disease Research, The Annual Review of Genomics and Human Genetics, 17, 2016, 131-54. https://doi.org/10.1146/annurev-genom-083115-022258

5. Magdalena Hryhorowicz, Daniel Lipin'ski, Joanna Zeyland, Ryszard Słomski, CRISPR/Cas9 Immune System as a Tool for Genome Engineering, Archivum Immunologiae et Therapiae Experimentalis, 65, 2017, 233-240 https://doi.org/10.1007/s00005-016-0427-5

6. Caroline Burgess, Victoria Cornelius, Sharon Love, Jill Graham, Michael Richards, Amanda Ramirez, Depression and anxiety in women with early breast cancer: five year observational cohort study, The British Medical Journal Papers, 330, 2005. https://doi.org/10.1136/bmj.38343.670868.D3

7. Sunil Mittala, Hardeep Kaura, Nandini Gautama, Anil K. Manthab, Biosensors for Breast Cancer Diagnosis: A Review of Bioreceptors, Biotransducers and Signal Amplification Strategies, Biosensors and Bioelectronics, 88, 2016, 217231. http://dx.doi.org/10.1016/j.bios.2016.08.028

8. Afsaneh Jalalian, Syamsiah B.T. Mashohor, Hajjah Rozi Mahmud, M. Iqbal B. Saripan, Abdul Rahman B. Ramli, Babak Karasfi, Computer-aided detection/diagnosis of breast cancer in mammography and ultrasound: a review, Clinical imagining, 37, 2013, 420-426. https://dx.doi.org/10.1016/j.clinimag.2012.09.024

9. Iraj Khalkhali, Ismael Mena, Linda Diggles, Review of imaging techniques for the diagnosis of breast cancer: a new role of prone scintimammography using technetium99m sestamibi, European Journal of Nuclear Medicine, 21, 1994, 357-362.

10. Hannah Frith, Diana Harcourt, Anna Fussell, Anticipating an altered appearance: Women undergoing chemotherapy treatment for breast cancer, European Journal of Oncology Nursing, 11, 2007, 385-391.
11. Dennis J. Slamon, Brian leyland-jones, Steven shak, Hank Fuchs, Virginia paton, Alex Bajamonde, Thomas Fleming, Wolfgang Eiermann, Janet Wolter, Mark Pegram, Jose Baslega, and Larry Norton, USE OF CHEMOTHERAPY PLUS A MONOCLONAL ANTIBODY AGAINST HER2 FOR METASTATIC BREAST CANCER THAT OVEREXPRESSES HER2, The New England Journal of Medicine, 344(1), 2001, 783792.

12. Gunter von Minckwitz, Michael Untch, Jens-Uwe Blohmer, Serban D. Costa, Holger Eidtmann, Peter A. Fasching, Bernd Gerber, Wolfgang Eiermann, Jo"rn Hilfrich, Jens Huober, Christian Jackisch, Manfred Kaufmann, Gottfried E. Konecny, Carsten Denkert, Valentina Nekljudova, Keyur Mehta, and Sibylle Loibl, Definition and Impact of Pathologic Complete Response on Prognosis After Neoadjuvant Chemotherapy in Various Intrinsic Breast Cancer Subtypes, Journal of Clinical Oncology, 30(15), 2012, 1796-1804.

13. Dawn L. Hershman, Theresa Shao, Lawrence H. Kushi, Donna Buono, Wei Yann Tsai, Louis Fehrenbacher, Marilyn Kwan, Scarlett Lin Gomez, Alfred I. Neugut, Early discontinuation and non-adherence to adjuvant hormonal therapy are associated with increased mortality in women with breast cancer, Breast Cancer Research and Treatment, 126, 2011, 529-537. https://doi.org/10.1007/s10549-010$\underline{1132-4}$

14. Dong Wang, Xian-Wang Wang, Xiao-Chun Peng, Ying Xiang, Shi-Bao Song, Ying-Ying Wang, Lin Chen, Victoria W. Xin, Yan-Ning Lyu, Jiafu Ji, Zhao-Wu Ma, Cheng-Bin Li, Hong-Wu Xin, CRISPR/Cas9 genome editing technology significantly accelerated herpes simplex virus research, Cancer Gene Therapy, 25, 2018, 93-105.

15. Kira S. Makarova, Daniel H. Haft, Rodolphe Barrangou, Stan J. J. Brouns, Emmanuelle Charpentier, Philippe Horvath, Sylvain Moineau, Francisco J. M. Mojica, Yuri I. Wolf, Alexander F. Yakunin, John van der Oost and Eugene V. Koonin, Evolution and classification of the CRISPR-Cas systems, NATURE REVIEW, 9, 2011, 467-477.

16. M. Niuz Morshed Khan, K. Khaldun Islam, A. Ashraf, N. Chandra Barman, A REVIEW ON GENOME EDITING BY CRISPR-CAS9 TECHNIQUE FOR CANCER TREATMENT, World cancer research journal, 7, 2020, e1510.

17. Si Chen, Heng Sun, Kai Miao, and Chu-Xia Deng, CRISPRCas9: from Genome Editing to Cancer Research, International Journal of Biological Sciences, 12(12), 2016, 1427-1436. https://doi.org/10.7150/ijbs.17421

18. Somali Chaterji, Eun Hyun Ahn, and Deok-Ho Kim, CRISPR Genome Engineering for Human Pluripotent Stem Cell Research, Theranostics, 7(18), 2017, 4445-4469. https://doi.org/10.7150/thno.18456

19. Lu Xiao-Jie, Xue Hui-Ying, Ke Zun-Ping, Chen Jin-Lian, Ji LiJuan, CRISPR-Cas9: a new and promising player in gene therapy, Journal of medical genetics, 52, 2015, 289-296. https://doi.org/10.1136/jmedgenet-2014-102968

20. Cem Kuscu, Mahmut Parlak, Turan Tufan, Jiekun Yang, Karol Szlachta, Xiaolong Wei, Rashad Mammadov \& Mazhar Adli, CRISPR-STOP: gene silencing through baseediting-induced nonsense mutations, Nature Methods, 14, 2017, 710-712. https://doi.org/10.1038/nmeth.4327 
21. Lucía Pérez, Erika Soto, Gemma Farre, Julia Juanos, Gemma Villorbina Ludovic Bassi, Vecente Medina, Antonio Jesús Serrat, Mariam Sahrawy Jose Antonio Rojas, Ignacio Romagosa, Pilar Muñoz, Changfu Zhu, Paul Christou, CRISPR/Cas9 mutations in the rice Waxy/GBSSI gene induce allelespecific and zygositydependent feedback effects on endosperm starch biosynthesis, Plant Cell Reports,38, 2019, 417-433.

22. Julian Grünewald, Ronghao Zhou, Sara P. Garcia, Sowmya Iyer, Caleb A. Lareau, Martin J. Aryee \& J. Keith Joung, Transcriptome-wide off-target RNA editing induced by CRISPR-guided DNA base editor, Research letters Nature, 569, 2019, 433-437.

23. Johanna F. Dekkers, James R. Whittle, François Vaillant, Huei-Rong Chen, Caleb Dawson, Kevin Liu, Maarten H. Geurts, Marco J. Herold, Hans Clevers, Geoffrey J. Lindeman, Jane E. Visvader, Modeling Breast Cancer Using CRISPR-Cas9-Mediated Engineering of Human Breast Organoids, Journal of the National Cancer Institute, 112(5), 2020, djz196. https://doi.org/10.1093/jnci/djz196

24. Rachel L. Mintz, Yeh-Hsing Lao, Chun-Wei Chi, Siyu He, Mingqiang Li, Chai Hoon Quek, Dan Shao, Boyuan Chen, Jing Han, Sihong Wang, Kam W. Leong, CRISPR/Cas9mediated mutagenesis to validate the synergy between PARP1 inhibition and chemotherapy in BRCA1-mutated breast cancer cells, Bioengineering and Translational Medicine, 5, 2020, e10152. https://doi.org/10.1002/btm.2.10152

25. Xueli Tian, Tingxuan Gu, Satyananda Patel, Ann M. Bode, Mee-Hyun Lee, and Zigang Dong, CRISPR/Cas9 - An evolving biological tool kit for cancer biology and oncology, npj Precision Oncology 8 Published in partnership with The Hormel Institute, University of Minnesota, 8, 2019.
26. Lu Xiao-Jie, Xue Hui-Ying, Ke Zun-Ping, Chen Jin-Lian, Ji LiJuan, CRISPR-Cas9: a new and promising player in gene therapy, Journal of Medical Genetics 52, 2015, 289-296, https://doi.org/10.1136/jmedgenet-2014-102968

27. Marta Martinez-Lage, Pilar Puig-Serra, Pablo Menendez, Raul Torres-Ruiz, and Sandra Rodriguez-Perales, CRISPR/Cas9 for Cancer Therapy: Hopes and Challenges, Biomedicines 6, 2018,105. https://doi.org/10.3390/biomedicines6040105

28. Debarati Ghosh, Prabhadevi Venkataramani, Saikat Nandi, and Sonali Bhattacharjee, CRISPR-Cas9 a boon or bane: the bumpy road ahead to cancer therapeutics, Cancer Cell International, $19, \quad 2019,2$ 12, https://doi.org/10.1186/s12935-019-0726-0

29. Chunyang Jiang, Lingxiang Meng, Bingjun Yang, Xin Luo, Application of CRISPR/Cas9 gene editing technique in the study of cancer treatment, Clinical Genetics, 97, 2020, 7388. https://doi.org/10.1111/cge.13589

30. Rodolphe Barrangou \& Jennifer A Doudna, Applications of CRISPR technologies in research and beyond, Nature biotechnology, 34(9), 2016, 933-941. https://doi.org/10.1038/nbt.3659

31. Lang $\mathrm{Yi}$, Jinming Li, CRISPR-Cas9 therapeutics in cancer: promising strategies and present challenges, Biochimica et Biophysica Acta, 1866, 2016, 197-207. https://dx.doi.org/10.1016/i.bbcan.2016.09.002

32. Yinnan Chen and Yanmin $Z$ hang, Application of the CRISPR/Cas9 System to Drug Resistance in Breast Cancer, Advanced science, 5: 1700964, 2018, 1-13. DOI: https://doi.org/10.1002/advs.201700964

Source of Support: None declared.

Conflict of Interest: None declared.

For any question relates to this article, please reach us at: editor@globalresearchonline.net New manuscripts for publication can be submitted at: submit@globalresearchonline.net and submit_ijpsrr@rediffmail.com 